\title{
Choosing Reporter-Quencher Pairs for Efficient Quenching Through Formation of Intramolecular Dimers
}

\author{
Mary Katherine Johansson
}

\begin{abstract}
Summary
Fluorescent energy transfer within dual-labeled oligonucleotide probes is widely used in assays for genetic analysis. Nucleic acid detection/amplification methods, such as real-time polymerase chain reaction, use dual-labeled probes to measure the presence and copy number of specific genes or expressed messenger RNA. Fluorogenic probes are labeled with both a reporter and a quencher dye. Fluorescence from the reporter is only released when the two dyes are physically separated via hybridization or nuclease activity. Fluorescence resonance energy transfer (FRET) is the physical mechanism that is most often cited to describe how quenching occurs.

We have found that many dual-labeled probes have enhanced quenching through a nonFRET mechanism called static quenching. Static quenching, which is also referred to as contact quenching, can occur even in "linear" oligonucleotide probes that have no defined secondary structure to bring the reporter and quencher pair into proximity. When static quenching accompanies FRET quenching, the background fluorescence of probes is suppressed. This chapter describes how to pair reporter and quencher dyes for duallabeled probes to maximize both FRET and static quenching. Data comparing various reporter-quencher pairs is presented as well as protocols for evaluation and optimization of the probes.
\end{abstract}

Key Words: FRET; intramolecular dimer; stacking interactions; fluorescent probes; biosensors.

\section{Introduction}

Fluorescent energy transfer within dual-labeled oligonucleotide probes is widely used in assays for genetic analysis. Nucleic acid detection/amplification methods, such as real-time polymerase chain reaction (PCR), use dual-

From: Methods in Molecular Biology, vol. 335:

Fluorescent Energy Transfer Nucleic Acid Probes: Designs and Protocols Edited by: V. V. Didenko (C) Humana Press Inc., Totowa, NJ 
labeled probes to measure the presence and copy number of specific genes or expressed messenger RNA. Fluorogenic probes such as TaqMan ${ }^{\circledR}$, molecular beacons, Amplifluors, and Scorpions are labeled with both a reporter and a quencher dye. Fluorescence from the reporter is only released when the two dyes are physically separated via hybridization or nuclease activity. Fluorescence resonance energy transfer (FRET) is the physical mechanism that is most often cited to describe how quenching occurs (1).

We have found that many dual-labeled probes have enhanced quenching through a non-FRET mechanism called static quenching. Static quenching, which is also referred to as contact quenching, operates through formation of a ground-state complex. The reporter and quencher dyes can bind together to form a reporter-quencher ground-state complex. The same forces that control dye aggregation presumably control this formation. When a ground-state complex forms the excited-state energy levels of the donor and quencher dyes couple giving the ground-state complex its own electronic properties, such as being nonfluorescent and having a unique absorption spectrum. A ground-state complex that is an intramolecular dimer can form even in "linear" oligonucleotide probes that have no defined secondary structure to bring the reporter and quencher pair into proximity. Figure 1 compares the static and FRET quenching mechanisms in "linear" reporter-quencher dual-labeled oligonucleotides.

When static quenching occurs accompanies FRET quenching, the background fluorescence of probes is suppressed $(2,3)$. Dual-labeled oligonucleotide probes that only have quenching via the FRET-quencher pairs are selected such that they can form a ground state complex, the signal/background ratios are much higher, up to a 10- to 30-fold increase in fluorescence signal, because of more efficient quenching.

This chapter describes how to pair reporter and quencher dyes for duallabeled probes to maximize both FRET and static quenching. Data comparing various reporter-quencher pairs is presented as well as protocols for evaluation and optimization of the probes. It is important to recognize that quenching efficiency can depend on many factors, including temperature and buffer composition. Therefore, quenching via intramolecular dimers is only one of many criteria that should be considered when designing dual-labeled probes.

\subsection{Important Probe Design Considerations}

\subsubsection{Positioning of Dye Labels}

Standard convention places the quencher on the 3 ' and the reporter on the 5 'end of the probe. This is primarily because in oligonucleotide synthesis, all failure sequence fragments contain only the 3 ' label. Therefore, if the quality of synthesis and/or purification is poor, there will be an excess of $3^{\prime}$-quencher- 
Static Quenching

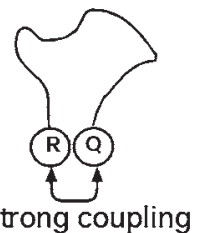

strong coupling

$\rightarrow$ physical association

produces ground state complex

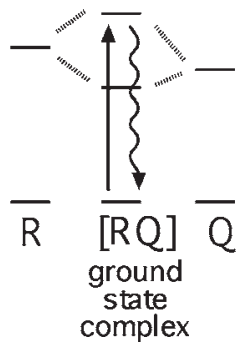

FRET Quenching
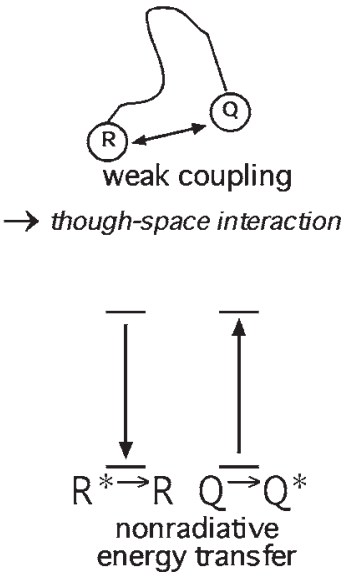

Fig. 1. Static and Förster resonance energy transfer quenching mechanisms in "linear" reporter-quencher dual-labeled oligonucleotides.

labeled oligos. This is a better alternative to having failure oligos containing only a 3'-reporter, which will raise the background fluorescence of the probe (4).

Dye labels may be available as a controlled pore glass (CPG), amidite, or active ester. The ease and yield of dye incorporation follows the order of CPG, amidite, ester, and this is generally reflected in probe price. A CPG dye is used to make 3' labels, whereas amidites and esters can be used for internal or 5' labels. Thus, to make a conventional 5'-reporter-3'-quencher probe, a reporteramidite and a quencher-CPG are used. In Table 1, reporter dyes that are available as amidites are shown in bold. In the manufacturing of dye-labeled oligonucletides, it is more convenient and cost-effective to use dye amidites rather than succinimdyl esters. However, not all dye labels can be prepared as phosphoramidites that can withstandard oligo synthesis conditions.

\subsubsection{Inherent Quenching by Bases}

Another important consideration in the placement of the reporter label is that the oligo bases, especially guanine, quench many fluorophores. Therefore, reporter dyes should not be placed directly next to $G$ residues $(5,6)$.

\section{Materials}

Table 1 is a listing of reporter dyes that are commonly used to label oligos. The dyes that are available as an amidite are shown in bold. Some of the dyes 
Table 1

Common Reporter Dyes for Oligos

\begin{tabular}{|c|c|c|c|}
\hline Dye name & Absorption max (nm) & Emission max (nm) & Dye type \\
\hline BODIPY FL & 502 & 510 & $\mathrm{O}$ \\
\hline FAM & 495 & 520 & $\mathbf{F}$ \\
\hline Oregon Green 488 & 494 & 517 & $\mathrm{~F}$ \\
\hline Rhodamine Green & 503 & 528 & $\mathrm{R}$ \\
\hline Oregon Green 514 & 506 & 526 & $\mathrm{~F}$ \\
\hline TET & 521 & 536 & $\mathbf{F}$ \\
\hline Cal Gold & 522 & 544 & $\mathbf{F}$ \\
\hline BODIPY R6G & 528 & 547 & $\mathrm{O}$ \\
\hline Yakima Yellow & 526 & 548 & $\mathbf{F}$ \\
\hline JOE & 520 & 548 & $\mathrm{~F}$ \\
\hline HEX & 535 & 556 & $\mathbf{F}$ \\
\hline Cal Orange & 540 & 561 & $\mathbf{R}$ \\
\hline BODIPY TMR-X & 544 & 570 & $\mathrm{O}$ \\
\hline Quasar-570/Cy3 & 550 & $\mathbf{5 7 0}$ & $\mathbf{C}$ \\
\hline TAMRA & 555 & 576 & $\mathbf{R}$ \\
\hline Rhodamine Red-X & 560 & 580 & $\mathrm{R}$ \\
\hline Redmond Red & 554 & 590 & $\mathbf{O}$ \\
\hline BODIPY 581/591 & 581 & 591 & $\mathrm{O}$ \\
\hline Cy3.5 & 581 & 596 & $\mathbf{C}$ \\
\hline $\mathrm{ROX}$ & 575 & 602 & $\mathrm{R}$ \\
\hline Cal Red/Texas Red & 593 & 613 & $\mathrm{R}$ \\
\hline BODIPY TR-X & 588 & 616 & $\mathrm{O}$ \\
\hline BODIPY 630/665-X & 647 & 665 & $\mathrm{O}$ \\
\hline Pulsar-650 & 460 & 650 & $\mathbf{O}$ \\
\hline Quasar-670/Cy5 & 649 & 670 & $\mathbf{C}$ \\
\hline Cy5.5 - - 2 & 675 & 694 & $\mathbf{C}$ \\
\hline
\end{tabular}

Dyes in bold are available as amidites and/or CPGs. F, fluorescein; R, rhodamine; $\mathrm{C}$, cyanine; O, other (see Fig. 2).

in Table $\mathbf{1}$ are proprietary and others, such as FAM, are ubiquitous. There are many commercial sources for fluorescent dye labels and for oligonucleotide probes. The core structures of the fluorescein, rhodamine, and cyanane dyes are shown in Fig. 2.

Table 2 lists dark quenchers that are commonly used in fluorogenic oligo probes. The quenchers that are available as an amidite as well as a CPG are shown in bold; these quenchers can easily be incorporated as 5', 3', or internal modifications. Many of the quenchers listed in Table 2 are proprietary, yet, they may be licensed and sold by several different companies. Figure 2 shows the core structures of dabcyl and Black Hole Quencher ${ }^{\mathrm{TM}}$ (BHQ). 
<smiles>O=C(O)C=CC(O)C=CC=CC=C1C=CC(=O)C=C1</smiles>

Fluorescein<smiles>[R][N+]([R])=CC=COC(=CC=C)C(=CC)C(=CC)C1=CC=CC(=O)C1</smiles>

Rhodamine<smiles>[R]N1c2ccccc2C(C)(C)C1C=CC1Nc2ccccc2C1(C)C</smiles>

Cyanine<smiles>[R]c1ccc(N)cc1</smiles>

Dabcyl<smiles>[R]CN([R])c1cccc(N=NC2C=CC(N=Nc3ccc([R6])cc3)=C2[R8])c1</smiles>

Fig. 2. Core structures for fluorescein, rhodamine and cyanine dyes, dabcyl, and Black Hole Quenchers ${ }^{\mathrm{TM}}$.

Table 2

Dark Quenchers for Oligonucleotide Reporters

\begin{tabular}{lc} 
Dye name & Absorption max (nm) \\
\hline Dabcyl & $\mathbf{4 5 3}$ \\
QSY 35 & 475 \\
BHQ-0 & $\mathbf{4 9 5}$ \\
Eclipse & $\mathbf{5 3 0}$ \\
BHQ-1 & $\mathbf{5 3 4}$ \\
QSY 7 & 560 \\
QSY 9 & 562 \\
BHQ-2 & $\mathbf{5 7 9}$ \\
ElleQuencher & 630 \\
Iowa Black & 651 \\
QSY 21 & 661 \\
BHQ-3 & $\mathbf{6 7 2}$ \\
\hline
\end{tabular}

Quenchers in bold are available in both CPG and amidite forms.

\section{Methods}

\subsection{Choice of Reporter-Quencher Pairs}

1. The first consideration in choosing a reporter dye is to check that the excitation and emission wavelengths are compatible with the instrumentation used to read 
fluorescence. For example, FAM is generally used with $488 \mathrm{~nm}$ light sources. There are now many choices for reporter dyes across the visible spectrum, and new products regularly appear (Table 1) (7). For multiplexing experiments, which use several reporter dyes to track different oligo sequences, the emission maxima of reporter dyes should be separated by at least $15 \mathrm{~nm}$ (see Notes $\mathbf{1}$ and 2).

2. There are a few criteria to keep in mind when choosing a quencher dye (Table 2). For FRET quenching, the absorption spectrum of the quencher must overlap with the fluorescence spectrum of the reporter. The first generation of dual-labeled oligo probes used the TAMRA dye (absorbance at $558 \mathrm{~nm}$ ) as a quencher for FAM (emission at $517 \mathrm{~nm}$ ). However, TAMRA has its own emission at $577 \mathrm{~nm}$ that can contribute to background fluorescence signal. Therefore, it is advantageous to use dark quenchers, such as the BHQs, which have no native fluorescence.

3. To pair reporters and quenchers for static quenching, one has to consider the structures of the dyes (Fig. 2). Static quenching involves formation of an intramolecular dimer; the dyes aggregate and stick together. Dye aggregation in aqueous solvents is controlled by electrostatic, steric, and hydrophobic forces (2).

4. Cyanine (Cy3, Quasar 670, and others) and rhodamine (Cal Orange, Cal Red, ROX, and so on) dyes are quite planar, hydrophobic, and have delocalized charge because of quaternary nitrogens. It has been observed that these dye structures undergo more static quenching with the BHQ dyes than fluoresceins (e.g., FAM, JOE, TET, Cal Gold, HEX) (Figs. 3-5).

\subsection{Measuring Quenching Efficiencies}

See Notes 3-5 and Fig. 6 for examples of assays measuring quenching efficiencies for probe evaluation. Experimental conditions, such as temperature and buffer composition, can dramatically affect quenching efficiency and dye fluorescence (see Notes 6 and 7).

\subsubsection{Sample Preparation and Analysis}

1. Probe concentrations in samples for fluorescence measurements should be less than $0.5 \mu M$. This is because fluorescence measurements can become distorted owing to the re-absorption of emitted light (8).

2. All fluorescence intensities should be corrected by subtracting the fluorescence intensity of a buffer blank.

3. Quenching data is often reported as a signal/background ratio where the background is from the intact (quenched) probe and the signal is recorded after hybridization or nuclease treatment (dequenched). Percentage quenching is calculated by dividing the signal of the dequenched probe (minus buffer blank) by the signal of the quenched probe (minus buffer blank), multiplying the result by 100 , and then subtracting the result from 100 .

\subsubsection{Hybridization Assay}

1. In a hybridization assay, it is important to use a buffer that contains $\mathrm{Mg}^{2+}$, such as PCR buffer (10 $\mathrm{m} M$ trizma hydrochloride, $50 \mathrm{mM} \mathrm{KCl}$, and $3.5 \mathrm{~m} M \mathrm{MgCl}_{2}$ ). 

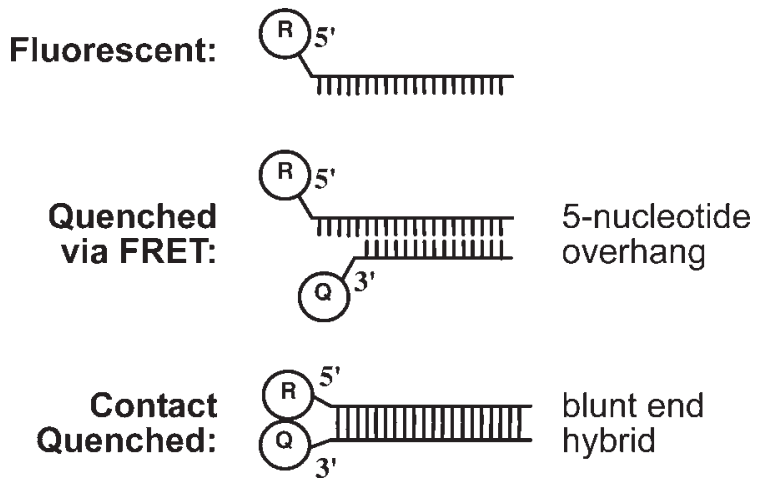

Fig. 3. Structures used by Marras et al. (5) to measure percentage quenching via Förster resonance energy transfer and contact quenching.

2. If a complementary sequence has extra bases on each end, the binding is stronger than if exact complement is used. (The complementary sequences used in Fig. 5 have three extra $\mathrm{T}$ bases on each end.)

3. A probe should be used that has a melting temperature above room temperature (see Note 8).

4. After adding a fivefold molar excess of complement, the fluorescence intensity should be monitored until it reaches a final value. This usually takes between 5 and $15 \mathrm{~min}$.

\subsubsection{Nuclease Digestion Assay}

1. There are several nucleases that can be used for digestion assays. Snake venom phosphodiesterase with bacterial alkaline phosphatase yield the nucleoside monomers through exonuclease digestion (9). DNase I and Bal 31 are both endonucleases that degrade both single and double-stranded DNA (USB, cat. no. 14367 and $70011 Y$ ).

2. The probe should be dissolved in the buffer for enzyme digestion and split into two fractions, one of which is a control that does not receive the digestion enzyme.

3. The extent of oligo digestion can be monitored by anion-exchange high-performance liquid chromatography.

4. After incubation, both the control and the digested sample should be diluted with buffer (e.g., PCR buffer) and the fluorescence intensities can be measured.

\subsubsection{Determination of Quenching Mechanism}

In order to distinguish definitively between FRET and static quenching it is necessary to measure fluorescence lifetimes. The fluorescence lifetime is the average time before a dye emits a photon after the dye has absorbed a photon.

Dynamic quenching, which includes FRET, decreases both fluorescence intensity and fluorescence lifetime of the reporter dye by the same factor. In 


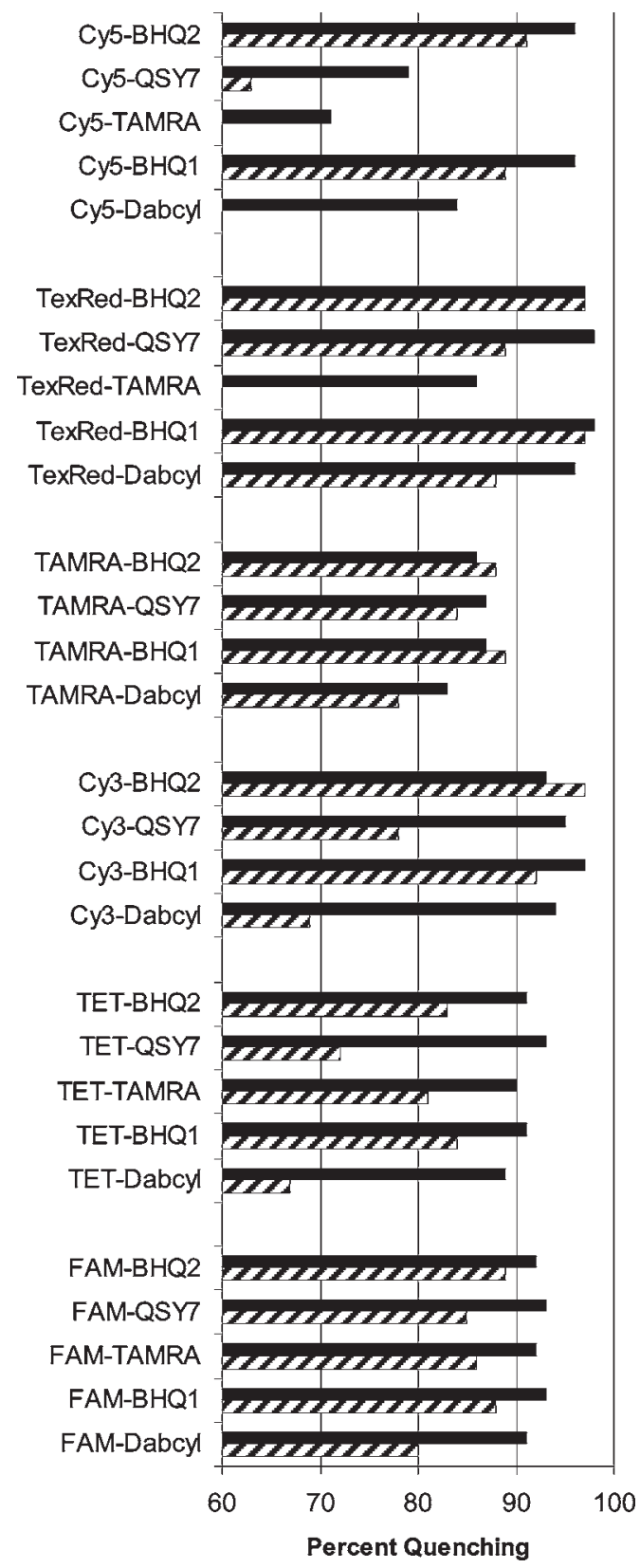

Fig. 4. Selected percentage quenching data from Marras et al. (5). Dark lines show contact quenching and striped lines show Förster resonance energy transfer quenching. 


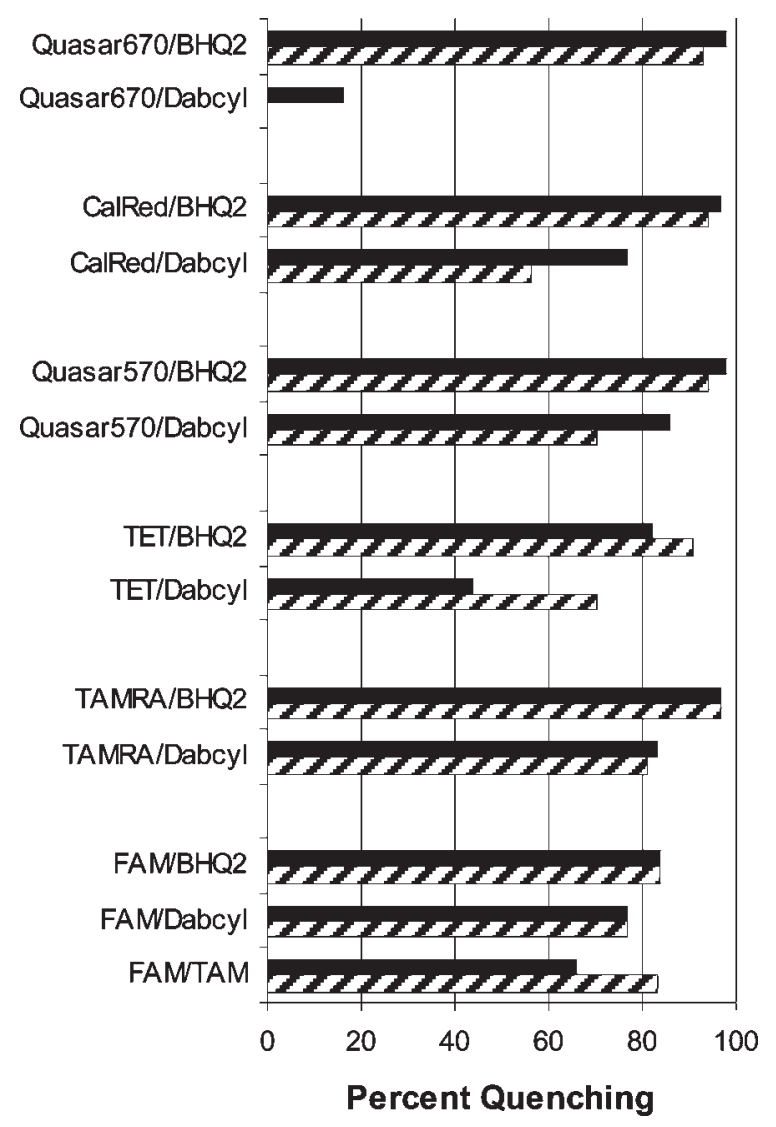

Fig. 5. Percentage quenching with dual-labeled $\beta$-actin probes. The $\beta$-actin sequence is 5'-d-ATG-CCC-TCC-CCC-ATG-CCA-TCC-TGC-G-3'. Dark lines show hybridization and striped lines nuclease digestion.

contrast, static quenching involves formation of a dye-quencher dimer. This effectively decreases the concentration of the fluorescent dye by creating a new, nonfluorescent reporter-quencher dimer. Because this dimer is formed before the dye absorbs a photon, static quenching does not change the reporter dye's fluorescence lifetime.

1. In a fluorogenic assay with a dual-labeled probe, if both the fluorescence intensity (I) and fluorescence lifetime $(\tau)$ change by the same amount going from the quenched to dequenched species, i.e., $\mathrm{I}_{\text {quenched }} / \mathrm{I}_{\text {dequenched }}=\tau_{\text {quenched }} / \tau_{\text {dequenched }}$, it can be concluded that dynamic quenching is the mechanism at work.

2. If $\tau_{\text {quenched }}=\tau_{\text {dequenched }}$ while $\mathrm{I}_{\text {quenched }} / \mathrm{I}_{\text {dequenched }}$ is less than one, static quenching is the mechanism at work. Another indication of static quenching is a change in the absorption spectrum of the probe in the quenched vs dequenched states (Fig. 7). 


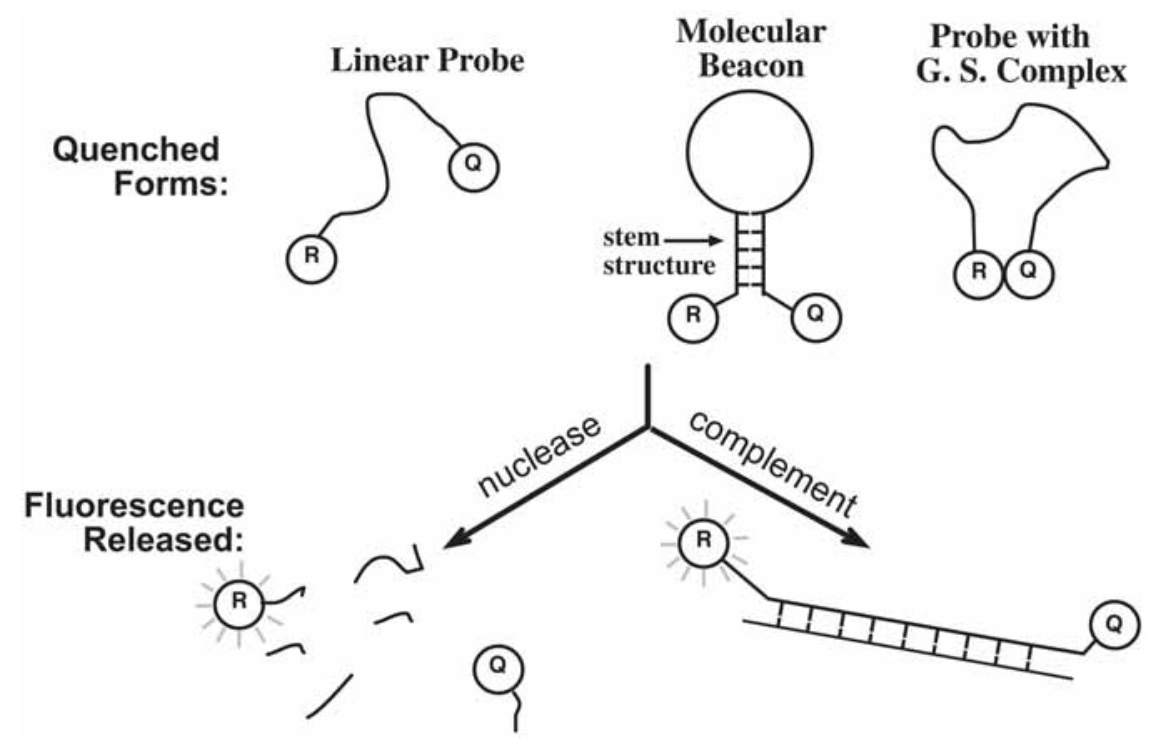

Fig. 6. Quenched probe structures and the release of fluorescence via digestion by nuclease and hybridization.

This is because the reporter-quencher dimer has its own unique absorption spectrum. FRET quenching does not effect the probe's absorption spectrum (2).

\section{Notes}

1. There are several commercial sources for fluorogenic oligonucleotide probes and some companies offer proprietary dyes. The BHQs, Quasar, and Cal Dyes were developed at Biosearch Technologies.

2. Most real-time PCR instruments use filters to selectively monitor reporter fluorescence. The ABI PRISM ${ }^{\circledR} 7700$ and 7900 perform spectral deconvolution. The instrument user manual may suggest combinations of reporters for multiplexing experiments.

3. There are several ways to screen combinations of reporter/quencher pairs. A series of quencher-reporter pairings were recently tested by Marras, Kramer, and Tyagi. They used complementary oligos with 5'-reporters and 3'-quenchers to bring the dyes directly together or at staggered distances to measure, respectively, contact-mediated and FRET quenching efficiencies (5) (Fig. 3). This method of bringing the dyes together in order to measure contact (static) quenching holds the reporter and quencher in a fairly constrained and fixed relative orientation. Furthermore, in this model, the reporter-quencher interaction may strongly depend on the length and rigidity of the oligo-dye linkers. Figure 4 shows the Marras et al. (5) quenching efficiencies for a series of reporter/quencher pairs with emission maxima spanning from 441 to $702 \mathrm{~nm}$. 


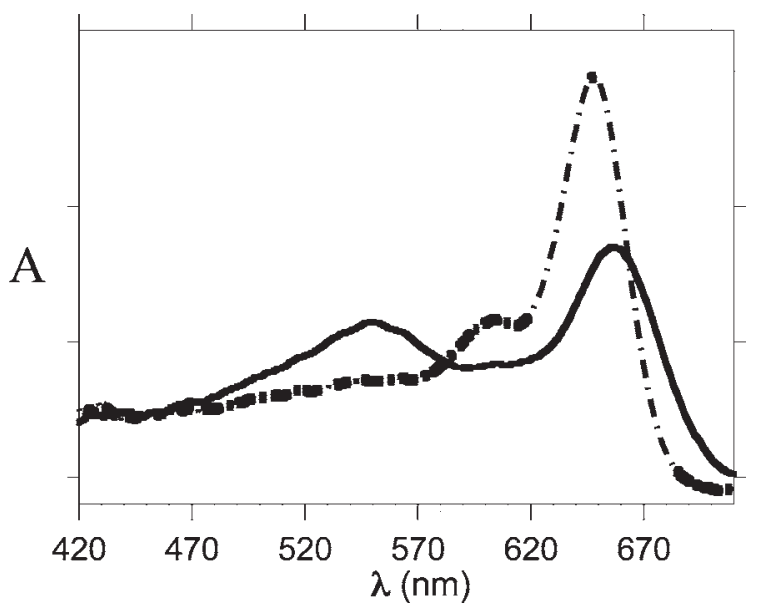

Fig. 7. Absorption spectrum of a Cy5/BHQ-1 probe alone (solid line) and hybridized to a complementary oligonucleotide (dashed line)

4. The data in Fig. $\mathbf{5}$ show percentage quenching that has been measured in a series of "linear" 5'-reporter-3'-quencher probes. The efficiency of static (also known as contact-mediated) quenching depends on the affinity of the reporter and quencher for each other (i.e., association constant). In a linear probe, the strength of this affinity is more critical than in a probe in which the reporter and quencher are held together through hybridization (10). Also, a linear probe can be thought of as a flexible linker that will allow the dyes to associate in a wider variety of conformations. Therefore, reporter-quencher pairs held in a fixed configuration, such as a molecular beacon or a hybrid, might have different quenching efficiencies than the same pair in a "linear" dual-labeled probe. These different structures are illustrated in Fig. 6. The quenching efficiency values measured by nuclease digestion differ from those measured via hybridization because the hybrid structure has an effect on the fluorescence intensities of many reporters (11). The 25mer linear probes with BHQ-2 as the quencher and Quasar-670, Cal Red, Quasar-570, and TAMRA as reporters all have quenching efficiencies greater than $90 \%$. Such high quenching efficiencies in "linear" probes are indicative of static quenching.

5. The BHQ are aromatic and quite hydrophobic (in fact, many dyes are only watersoluble after conjugation to oligos). One might expect that a hydrophobic reporter dye should be used to increase reporter-BHQ association. Some reporter dyes have phosphonate or sulfonates appended in order to increase water solubility. However, there is not very much data on intramolecular heterodimers with water-soluble dyes. Surprisingly, a sulfonated Cy 3 (Amersham Biosciences, cat. no. PA13101)/BHQ-2 $\beta$-actin probe has a quenching efficiency of $93 \%$ on both hybridization and nuclease digestion. Changes in the absorption spectra suggest formation of a reporter-quencher intramolecular dimer. 
6. Quenching efficiency within dual labeled probes can be temperature dependent. Association between the reporter and quencher that controls formation of the intramolecular dimer decreases with increasing temperature. Thus, efficient static (or contact) quenching at room temperature may significantly decrease at higher temperatures.

7. The fluorescence quantum yields of some dyes, such as the cyanines, decrease significantly with increasing temperature. Fluorescence intensity can also depend on $\mathrm{pH}$ and the local environment of the dye (12).

8. If a reporter-quencher pair that form a strong ground state complex are used in a molecular beacon (or other type of self-hybridizing probe), the additional stabilization owing to the reporter-quencher association can inhibit the molecular beacon from hybridizing to the complementary sequence at room temperature.

\section{Acknowledgments}

The author wishes to thank her colleagues at Biosearch Technologies. Financial support from Small Business Innovative Research grant 1R43GM60848 is gratefully acknowledged.

\section{References}

1. Didenko, V. V. (2001) DNA probes using fluorescence resonance energy transfer (FRET): designs and applications. Biotechniques 31, 1106-1121.

2. Johansson, M. K., Fidder, H., Dick, D., and Cook, R. M. (2002) Intramolecular dimers: a new strategy to fluorescence quenching in dual-labeled oligonucleotide probes. J. Am Chem. Soc. 124, 6950-6956.

3. Johansson, M. K. and Cook, R. M. (2003) Intramolecular dimers: a new design strategy for fluorescence-quenched probes. Chem. Eur. J. 9, 3466-3471.

4. Rudert, W. A., Braun, E. R., Faas, S. J., Menon, R., Jaquins-Gerstl, A., and Trucco, M. (1997) Double labeled fluorescent probes for 5' nuclease assays: purification and performance evaluation. Biotechniques 22, 1140-1145.

5. Marras, S. A. E., Russell, F. R., and Tyagi, S. (2002) Efficiencies of fluorescence resonance energy transfer and contact-mediated quenching in oligonucleotide probes. Nucliec Acids Res. 30, e122.

6. Seidel, C. A. M., Schulz, A., and Sauer, M. H. H. (1996) Nucleobase-specific quenching of fluorescent dyes. 1. Nucleobase one-electron redox potentials and their correlation with static and dynamic quenching efficiences. J. Phys. Chem. 100, 5541-5553.

7. Haugland, R. P. (2002) Handbook of Fluorescent Probes and Research Products, Molecular Probes, Eugene, OR.

8. Lakowicz, J. (1999) Principles of Fluorescence Spectroscopy, Plenum, New York.

9. Andrus, A. and Kuimelis, R. G. (2000) Current Protocols in Nucleic Acid Chemistry. Wiley and Sons, NY.

10. Bernacchi, S. and Mély, Y. (2001) Excitation interaction in molecular beacons: a sensitive sensor for short range modifications of the nucleic acid structure. Nucleic Acids Res. 29, e62. 
11. Crockett, A. O. and Wittwer, C. T. (2001) Fluorescein-labeled oligonucleotides for real-time PCR: using the inherent quenching of deoxyguanosine nucleotides. Anal. Biochem. 290, 89-97.

12. Sjöback, R., Nygren, J., and Kubista, M. (1998) Characterization of fluoresceinoligonucleotide conjugates and measurements of local electrostatic potential. Biopolymers 46, 445-453. 